\title{
ESTABILIZAÇÃO GLOBAL DO SISTEMA CAÓTICO DE LORENZ ATRAVÉS DO CONTROLE POR MODOS DESLIZANTES VIA OBSERVADORES DA NORMA
}

\author{
Victor Hugo Pereira Rodrigues*, Tiago Roux Oliveira* \\ *Departamento de Engenharia Eletrônica e Telecomunicações - Faculdade de Engenharia \\ Universidade do Estado do Rio de Janeiro - Rua São Francisco Xavier 524, sala 5001E - 20559-900 \\ Emails: victor.vhpr@yahoo.com, tiagoroux@uerj.br
}

\begin{abstract}
In this paper, we have assumed that all parameters of the Lorenz system are unknown and only the output variable is available for feedback. Exploring ISS (Input-to-State Stability) properties of the system, an upper bound for the norm of the unmeasured state vector is developed from the system output. Such norm estimate provided by the norm observer is applied to obtain the output-feedback sliding mode control law. Based on Lyapunov's stability theory, it was possible to guarantee that the proposed controller is capable to globally stabilize the Lorenz system. Simulation results are also presented to show the robustness of the control strategy with respect to parametric uncertainties.
\end{abstract}

Keywords - Chaotic Dynamics, Nonlinear Control, Global Stability, Output-feedback, Lorenz System

Resumo- Neste artigo, assume-se que todos os parâmetros do sistema de Lorenz são desconhecidos e que apenas uma variável de saída está disponível para o projeto do controlador. Explorando a característica ISS (Input-to-State Stability) do sistema, desenvolve-se um limitante superior para a norma do vetor de estado através desta única saída medida. Esse limitante fornecido pelo observador da norma é utilizado na lei de controle por modos deslizantes via realimentação de saída. Baseado na teoria de estabilidade de Lyapunov, foi possível mostrar que o controlador proposto é capaz de estabilizar globalmente o sistema de Lorenz. Resultados de simulações são apresentados para mostrar também a robustez da estratégia de controle a incertezas paramétricas.

Palavras-chave- Dinâmica Caótica, Controle Não-linear, Estabilidade Global, Realimentação de Saída, Sistema de Lorenz

\section{Introdução}

Sistemas caóticos têm sua existência conhecida desde o século XIX, no entanto sua importância para uma grande variedade de aplicações só começou a ser estudada nas últimas décadas. Sistemas dinâmicos são chamados de caóticos se suas órbitas são tipicamente não periódicas, limitadas dentro de uma região no espaço de estados e possuem alta sensibilidade às condições iniciais. A teoria caótica tem sido aplicada a vários campos de estudo como cardiologia (Cheng-Yu Yeh and Yau, 2012), circuitos eletrônicos (Guo and Liu, 2011), sincronização (Souza et al., 2008; Souza et al., 2012), comunicação segura (Cuomo and Oppenheim, 1993; Mozelli et al., 2007), conversão de energia (Hou, 2012), dinâmica dos fluidos (Lorenz, 1963) e economia (Goodwin, 1990).

Depois do trabalho pioneiro de (Ott et al., 1990), o controle de sistemas caóticos tem sido estudado de forma intensiva. Em (Ott et al., 1990), o método OGY foi desenvolvido permitindo converter um atrator caótico em um grande número de trajetórias periódicas instáveis injetando pequenas perturbações variáveis no tempo em um dos parâmetros acessíveis do sistema.

Em (Wang and Ge, 2001), uma técnica para eliminar os termos cruzados do sistema de Lorenz foi utilizada para desenvolver um controlador que estabiliza globalmente o sistema, no entanto o controlador necessita o conhecimento de todo o vetor de estado. Em (Araujo and Singh, 2002), um controlador via realimentação de saída por estrutura variável foi obtido, porém o sinal de controle era demasiadamente grande e o resultado de estabilidade é válido apenas localmente. Em (Guo and Liu, 2011), dois controladores são desenvolvidos, mas nenhum deles é robusto a incertezas.

Parte da teoria moderna de projeto de controle não-linear desenvolvida até o momento é baseada na hipótese do conhecimento das variáveis de estado do sistema. Entretanto, sabe-se, que essa hipótese não é válida na prática, pois em geral é possível medir apenas parte das variáveis do vetor de estado devido a limitações de número e localização de sensores. As demais variáveis não são fisicamente ou economicamente disponíveis. No caso de sistemas aproximáveis por um modelo dinâmico linear, é possível utilizar estimadores de estado. Ainda assim, é necessário um conhecimento razoável do modelo do processo, o que não é possível no caso de sistemas incertos (sistemas sujeitos a incertezas paramétricas, dinâmicas não modeladas e distúrbios externos). Os argumentos acima reforçam a necessidade do desenvolvimento de estratégias de controle de sistemas incertos baseadas apenas em informações da saída medida.

A contribuição deste artigo é a proposta de uma nova estratégia de controle para o sistema de Lorenz baseada na teoria de controle por modos deslizantes (Utkin, 1978). Para isso, assume-se que os parâmetros do sistema são desconhecidos e incertos. O controlador desenvolvido é capaz de garantir estabilidade global ao sistema caótico de Lorenz controlado utilizando apenas realimentação de saída. Esse resultado teórico estava em 
aberto na literatura e o presente trabalho propõe uma solução para este problema.

\section{Notação e Terminologia}

As seguintes notações e conceitos básicos são empregados ao longo do texto. A norma Euclidiana de um vetor $x$ e a correspondente norma induzida de uma matriz $A$ são denotadas por $|x|$ e $|A|$, respectivamente. Aqui, adotou-se a definição de (Filippov, 1964) para a solução de equações diferenciais com lado direito descontínuo. O conceito de estabilidade ISS (Input-to-State-Stability), assim como as definições de funções de classe $\mathcal{K}$ e $\mathcal{K}_{\infty}$ encontram-se de acordo com (Khalil, 2002):

Definição 1 Uma função contínua $\alpha:[0, a) \rightarrow[0, \infty)$ é dita pertencer à classe $\mathcal{K}$ se ela for estritamente crescente e $\alpha(0)=0$. Ela é dita pertencer à classe $\mathcal{K}_{\infty}$ se $\alpha=\infty$ e $\alpha(r) \rightarrow \infty \operatorname{com} r \rightarrow \infty$.

Definição $\mathbf{2} U m a$ função contínua $\beta \quad:[0, a) \times[0, \infty) \rightarrow[0, \infty)$ é dita pertencer à uma classe $\mathcal{K} \mathcal{L}$ se, para cada s fixo, o mapeamento $\beta(r, s)$ pertencer à classe $\mathcal{K}$ com respeito a $r$ e, para cada $r$ fixo, o mapeamento $\beta(r, s)$ é decrescente com respeito a $s$ e $\beta(r, s) \rightarrow 0$ com $s \rightarrow \infty$.

Definição 3 Considere o sistema $\dot{x}=f(t, x, u)$, onde $f:[0, \infty) \times \mathbb{R}^{n} \times \mathbb{R} \rightarrow \mathbb{R}^{n}$ é contínuo por partes em $t$ e localmente Lipschitz em $x$ e u. $O$ sistema é dito ser input-to-state stable (ISS) se existe uma função $\beta$ de classe $\mathcal{K} \mathcal{L}$ e uma função $\gamma$ de classe $\mathcal{K}$ tal que para qualquer estado inicial $x\left(t_{0}\right)$ e qualquer entrada limitada $u(t)$, a solução $x(t)$ existe para todo $t \geq t_{0}$ e satisfaz

$$
|x(t)| \leq \beta\left(\left|x\left(t_{0}\right)\right|, t-t_{0}\right)+\gamma\left(\sup _{t_{0} \leq \tau \leq t}|u(\tau)|\right) .
$$

Se na Definição 3 a função de classe $\mathcal{K} \mathcal{L}$ é uma exponencial, então a estabilidade ISS é do tipo exponencial.

\section{O Sistema de Lorenz}

O Sistema de Lorenz é um paradigma na área de sistemas caóticos visto que ele captura muitos aspectos da dinâmica caótica. Este sistema é uma simplificação das equações diferenciais parciais do trabalho de (Saltzman, 1962) que é constituído por três equações diferencias não-lineares com dinâmica dada por:

$$
\begin{aligned}
& \dot{x_{1}}=-\sigma x_{1}+\sigma x_{2} \\
& \dot{x_{2}}=r x_{1}-x_{1} x_{3}-x_{2} \\
& \dot{x_{3}}=-b x_{3}+x_{1} x_{2}
\end{aligned}
$$

em que $x_{1}, x_{2}, x_{3} \in \mathbb{R}$, formando o vetor de estado $x=\left[\begin{array}{lll}x_{1} & x_{2} & x_{3}\end{array}\right]^{T}$ e os parâmetros são $\sigma, r, b>0$.

As Figuras 1 e 2 ilustram a representação do sistema de Lorenz no espaço de estado e o comportamento das variáveis de estado em relação ao tempo de acordo com as equações (1)-(3). Nas simulações, os parâmetros e condições iniciais foram escolhidos como: $\sigma=10, r=28, b=\frac{8}{3}, \delta=0.1$, $x_{1}(0)=x_{3}(0)=0$ e $x_{2}(0)=1$.

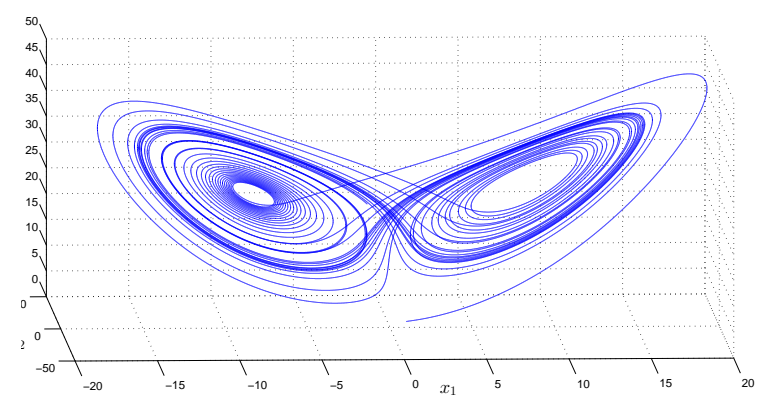

Figura 1: Representação do sistema de Lorenz no espaço de estado.

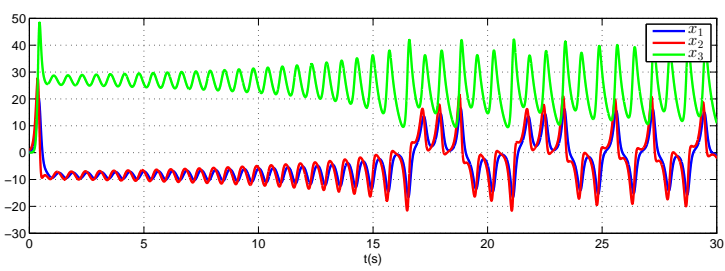

Figura 2: Sensibilidade às condições iniciais: comportamento das variáveis de estado.

\section{Controlando o Sistema de Lorenz: Realimentação de Estado}

Com o uso do controle por modos deslizantes forçar-se-a que o sistema de Lorenz, dada uma condição inicial arbitrária, alcance e, posteriormente, se mantenha em uma superfície prédefinida dentro do espaço de estados, conhecida como superfície de deslizamento. Com este método de controle obtém-se duas vantagens: a redução de ordem do sistema e a robustez em relação às incertezas (Utkin, 1978). Além disso, o sinal de controle será colocado apenas em um dos canais do sistema, utilizando uma função de Lyapunov da forma $V(s)=\frac{s^{2}}{2}$, tal que a variável de deslizamento $s$ é definida pela combinação linear dos estados:

$$
s(x)=a_{1} x_{1}+a_{2} x_{2}+a_{3} x_{3},
$$

em que $a_{1}, a_{2}$ e $a_{3}$ são constantes apropriadas.

Para garantir a condição de deslizamento ideal, segundo os critérios estabelecidos pela teoria de Lyapunov, deve-se fazer com que a órbita do 
sistema mantenha-se sobre o ponto de mínimo da função $V(s)$. Quando $s=0$, a trajetória já está na superfície de deslizamento, então nosso problema consiste em manter as trajetórias do sistema em $s=0$.

Assim sendo, colocando o sinal de controle $u$ no segundo canal do sistema, tem-se:

$$
\begin{aligned}
& \dot{x_{1}}=-\sigma x_{1}+\sigma x_{2} \\
& \dot{x_{2}}=r x_{1}-x_{1} x_{3}-x_{2}+u \\
& \dot{x_{3}}=-b x_{3}+x_{1} x_{2} .
\end{aligned}
$$

Definição 4 Se a solução do sistema (5)-(7) representada por $x(t)$ satisfaz

$$
s \dot{s}<0,
$$

então um modo deslizante ideal ocorrerá assintoticamente em $s(x)=0$. Por outro lado, se

$$
s \dot{s} \leq-\delta|s|, \text { para } \delta>0,
$$

então o modo deslizante ideal ocorrerá em tempo finito (Utkin et al., 1999, seção 2.5).

Teorema 1 Se o controlador por modos deslizantes $u$ é dado por

$$
u=-\left[\left|x_{1} x_{3}\right|+\left|r x_{1}\right|+\left|x_{2}\right|+\delta\right] \operatorname{sgn}\left(x_{2}\right),
$$

onde $\delta$ é uma constante positiva arbitrariamente pequena, então o ponto de equilíbrio $\left(x_{1}, x_{2}, x_{3}\right)=(0,0,0)$ do sistema dado pelas equações (5)-(7) é globalmente assintoticamente estável.

Prova: Considere a variável de deslizamento

$$
s(x)=x_{2} .
$$

Construindo uma função de Lyapunov como

$$
V=\frac{1}{2} x_{2}^{2},
$$

então, a derivada de $V$ ao longo da trajetória do sistema formado pelas equações (5), (6) e (7) é dada por

$$
\begin{aligned}
\dot{V} & =x_{2} \dot{x}_{2} \\
& =x_{2}\left(r x_{1}-x_{1} x_{3}-x_{2}+u\right) .
\end{aligned}
$$

A partir da equação (13), é fácil constatar que $\dot{V}<0$ se $x_{2} \dot{x}_{2}<0$. Suponha que o controlador por modos deslizantes tenha a seguinte forma:

$$
u=-\rho(x) \operatorname{sgn}(s) \text {. }
$$

Fazendo-se

$$
d(x)=r x_{1}-x_{1} x_{3}-x_{2},
$$

pode-se reescrever a equação (14) como

$$
\dot{V}=x_{2}[d(x)+u] \text {, }
$$

em que,

$$
\begin{aligned}
|d(x)| & =\left|r x_{1}-x_{1} x_{3}-x_{2}\right| \\
& \leq\left|r x_{1}\right|+\left|x_{1} x_{3}\right|+\left|x_{2}\right|
\end{aligned}
$$

projetando o controlador dado pela equação (15) de tal forma que $\rho(x)>|d(x)|$ garante-se que $\dot{V}<0, \forall x$, depois de um certo intervalo de tempo. Logo,

$$
\rho(x)=\left|r x_{1}\right|+\left|x_{1} x_{3}\right|+\left|x_{2}\right|+\delta,
$$

garante-se que o modo deslizante ideal seja atingido em tempo finito. Desta forma, o controlador pode ser descrito como

$$
u=-\left[\left|r x_{1}\right|+\left|x_{1} x_{3}\right|+\left|x_{2}\right|+\delta\right] \operatorname{sgn}\left(x_{2}\right),
$$

da mesma forma que em (10). Ao substituir (21) em (17), obtêm-se

$$
\dot{V} \leq-\delta x_{2} \operatorname{sgn}\left(x_{2}\right)=-\delta\left|x_{2}\right| .
$$

De acordo com a equação (22), a superfície de deslizamento $s=x_{2}=0$ é alcançada em tempo finito. A partir da Definição 3, pode-se verificar que os subsistemas (5) and (7) são ISS com respeito a $x_{2}$ e que, consequentemente, $x_{1}$ e $x_{3}$ tendem a zero exponencialmente. Assim sendo, o sistema em malha fechada é globalmente assintoticamente estável.

A lei de controle acima é capaz de estabilizar o sistema, no entanto precisar-se conhecer todas as variáveis de estado e, no mínimo, o parâmetro $r$ ou um limitante superior para o mesmo. A Figura 3 mostra um diagrama de blocos que ilustra o controlador baseado em realimentação de estado descrito acima.

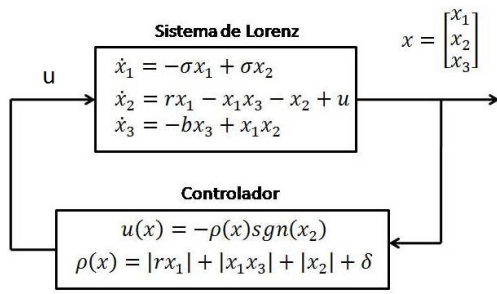

Figura 3: Diagrama de blocos do controle por realimentação de estado.

Neste trabalho, assume-se que todos os parâmetros do sistema de Lorenz são incertos. Nas seções a seguir propõe-se uma estratégia de controle utilizando limitantes dos parâmetros do sistema e sem o conhecimento exato destes. Além disso, utiliza-se apenas o valor medido de uma das variáveis de estado como saída para o projeto do controlador. 


\section{Observadores da Norma}

O observador da norma é uma técnica capaz de estimar a norma do vetor de estado de um sistema através de valores de limitantes inferiores e superiores de seus parâmetros. Essa estimativa estará sempre acima da norma do estado observado a menos de um termo exponencial decrescente, relacionado com o transitório da resposta.

Em (Oliveira et al., 2010), foi desenvolvida um método baseado nos trabalhos de (Sontag and Wang, 1997) e (Krichman et al., 2001) para a obtenção dos observadores da norma. Este método é capaz de contemplar de forma direta as equações diferenciais que formam o sistema de Lorenz. A descrição do método com maior rigor matemático pode ser encontrada nas referências que foram citadas acima, e os principais resultados são vistos abaixo.

Seja um sistema não-linear genérico:

$$
\dot{x}=f(t, x, y)
$$

onde $y \in \mathbb{R}$ é encarado como uma entrada para o sistema e $x \in \mathbb{R}^{n}$ é o estado do sistema, não disponível. As funções incerta $f$ é contínua por partes em t e localmente Lipschitz contínua nos outros argumentos.

Definição 5 Um observador da norma para o subsistema (23) é um sistema dinâmico SISO de primeira ordem da forma:

$$
\dot{\hat{x}}=-\lambda \hat{x}+\varphi(y, t),
$$

com entrada $\varphi(y, t)$ e saída $\hat{x}$, tal que o estado $x$ de (23) satisfaz

$$
|x(t)| \leq|\hat{x}(t)|+\bar{k}(|\hat{x}(0)|+|x(0)|) e^{-\lambda t},
$$

$\forall t \geq 0$, com alguma constante $\bar{k}>0$ e para cada estado inicial $x(0)$ e $\hat{x}(0)$, sendo: (i) $\lambda>0$ uma constante e (ii) $\varphi(y, t)$ uma função não-negativa, contínua em $y$ e continua por partes em $t$, satisfazendo $\varphi \leq \Psi(|y|)+k$, para algum $\Psi \in \mathcal{K} e$ alguma constante $k \geq 0$.

No caso invariante no tempo, sabe-se que o sistema (23) é ISS com respeito a $y$, então ele admite tal observador da norma e a planta é de fase mínima.

Por análise direta do sistema (5)-(7) é fácil visualizar que a dinâmica que governa $x_{1}$ é ISS em relação a $x_{2}$ e que a dinâmica de $x_{3}$ é ISS com relação a função $x_{1} x_{2}$. Assim sendo, ao considerar a saída do sistema $y=x_{2}$, pode-se utilizar a técnica dos observadores da norma para construir um controlador baseado apenas em realimentação de saída.

Deste modo, define-se os limitantes dos parâ- metros do sistema de Lorenz da seguinte forma:

$$
\begin{gathered}
\underline{\sigma} \leq \sigma \leq \bar{\sigma} \\
\underline{r} \leq r \leq \bar{r} \\
\underline{b} \leq b \leq \bar{b}
\end{gathered}
$$

e, então pode-se especificar o observador da norma para as variáveis de estado $x_{1}$ e $x_{3}$, como:

$$
\begin{aligned}
& \dot{\hat{x}}_{1}=-\underline{\sigma} \hat{x}_{1}+\bar{\sigma} y \\
& \dot{\hat{x}}_{3}=-\underline{b} \hat{x}_{3}+\hat{x}_{1} y
\end{aligned}
$$

onde $\hat{x}_{1}, \hat{x}_{3} \in \mathbb{R}$ são incluídos no vetor de estado estimado $\hat{x}=\left[\begin{array}{lll}\hat{x}_{1} & y & \hat{x}_{3}\end{array}\right]^{T}$ e os limitantes $\underline{\sigma}, \bar{\sigma}, \underline{r}, \bar{r}$, $\underline{b}, \bar{b}$ são constantes positivas conhecidas.

Assim como em (25), majorantes para a norma de $x_{1}$ e $x_{3}$ podem ser obtidos a menos de termos exponencialmente decrescentes devido às condições iniciais $x_{1}(0)$ e $x_{3}(0)$, tratados aqui como termos transitórios.

\section{Controle por Modos Deslizantes Via Realimentação de Saída}

A partir dos resultados das seções anteriores é possível desenvolver uma lei de controle por realimentação de saída baseada em observadores da norma que garanta a estabilidade global do sistema de Lorenz.

Teorema 2 Se controlador por modos deslizantes via realimentação de saída u é dado por

$$
u=-\left[\left|\bar{r} \hat{x}_{1}\right|+\left|\hat{x}_{1} \hat{x}_{3}\right|+|y|+\delta\right] \operatorname{sgn}(y),
$$

onde $\delta$ é uma constante positiva arbitrariamente pequena e $y=x_{2}$ é a única saída medida do sistema (5)-(7), então o ponto de equilíbrio $\left(x_{1}, x_{2}, x_{3}, \hat{x}_{1}, \hat{x}_{3}\right)=(0,0,0,0,0)$ do sistema dado pelas equações (5)-(7) e (29)-(30) é globalmente assintoticamente estável.

Prova: Suponha o sistema formado pelas equações (5)-(7), e o observador da norma construído a partir da saída $y=x_{2}$, dado pelas equações (29)-(30). Considere a variável de deslizamento

$$
s(x):=x_{2}=y
$$

Construindo uma função de Lyapunov como

$$
V=\frac{1}{2} y^{2}
$$

então, a derivada de $V$ ao longo da trajetória do sistema formado pelas equações (5)-(7) é dada por

$$
\begin{aligned}
\dot{V} & =y \dot{y} \\
& =y\left(r x_{1}-x_{1} x_{3}-y+u\right) .
\end{aligned}
$$

A partir da equação (34) é fácil constatar que $\dot{V}<0$ se $y \dot{y}<0$. 
Suponha um controlador por modos deslizantes via realimentação de saída da forma:

$$
u=-\rho(\hat{x}) \operatorname{sgn}(y) .
$$

Fazendo-se

$$
d(x)=r x_{1}-x_{1} x_{3}-y
$$

pode-se reescrever a equação (35) como

$$
\dot{V}=y[d(x)+u] \text {, }
$$

em que

$$
|d(x)| \leq\left|\bar{r} \hat{x}_{1}\right|+\left|\hat{x}_{1} \hat{x}_{3}\right|+|y| .
$$

Projetando o controlador (36) de tal forma que que $\rho(\hat{x})>|d(x)|$ garante-se que $\dot{V}<0, \forall x$, depois de um regime transitório. Pode-se fazer então:

$$
\rho(\hat{x})=\left|\bar{r} \hat{x}_{1}\right|+\left|\hat{x}_{1} \hat{x}_{3}\right|+|y|+\delta,
$$

garantindo que o modo deslizante ideal seja atingido em tempo finito. Dessa forma, o controlador (36) pode ser descrito como

$$
u=-\left[\left|\bar{r} \hat{x}_{1}\right|+\left|\hat{x}_{1} \hat{x}_{3}\right|+|y|+\delta\right] \operatorname{sgn}(y) .
$$

assim como em (31). Substituindo (41) em (38) garante-se que

$$
\dot{V} \leq-\delta|y|
$$

Então, de acordo com a Definição $4, y=x_{2}=0$ é alcançada em tempo finito e, de acordo com a propriedade ISS das dinâmica de $x_{1}, x_{3}, \hat{x}_{1}$ e $\hat{x}_{2}$ com respeito a $y$, todos os estados tendem globalmente exponencialmente a zero.

A Figura 4 apresenta um diagrama de blocos que ilustra a ação do controlador por realimentação de saída.

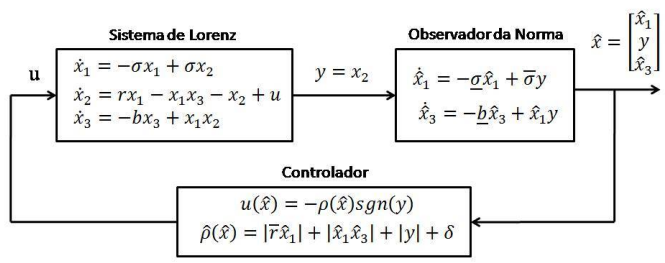

Figura 4: Diagrama de blocos: controle por realimentação de saída.

\section{Resultados Numéricos}

Em todas as simulações os parâmetros e condições iniciais foram escolhidos como: $\sigma=10, r=28$, $b=\frac{8}{3}, \delta=0.1, x_{1}(0)=x_{3}(0)=0$ e $x_{2}(0)=1$. A estimativas para os parâmetros foram escolhidas como $\underline{\sigma}=5, \bar{\sigma}=15, \underline{r}=14, \bar{r}=42$, $\underline{b}=\frac{4}{3}$ e $\bar{b}=\frac{16}{3}$. Além de escolher $\delta=0.1 \mathrm{e}$ $\hat{x}_{1}(0)=\hat{x}_{2}(0)=\hat{x}_{3}(0)=0$. As Figuras 5 e 6 mostram, em malha aberta, as normas das variáveis de estados e suas respectivas normas observadas de acordo com as equações (29) e (30). A Figura 7 mostra o funcionamento do sinal de controle em função do tempo. As Figuras 8 e 10 apresentam novamente as normas das variáveis de estados e suas respectivas normas observadas, em malha fechada. A Figura 9 ilustra o comportamento das variáveis de estado em malha fechada.

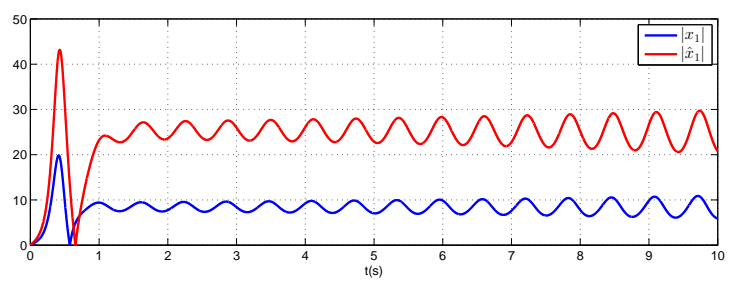

Figura 5: Comportamento da norma de $x_{1}$ e de $\hat{x}_{1}$, em malha aberta.

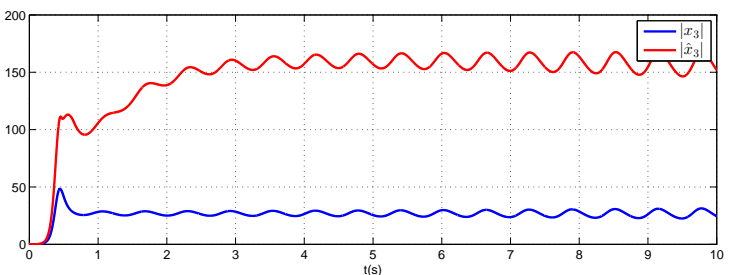

Figura 6: Comportamento da norma de $x_{3}$ e de $\hat{x}_{3}$, em malha aberta.

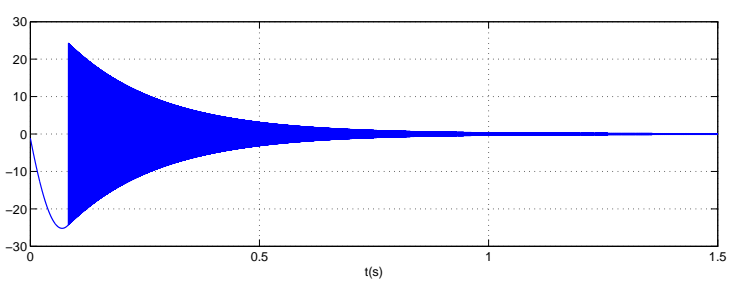

Figura 7: Comportamento do controlador u.

\section{Conclusão}

Explorando as características ISS do sistema de Lorenz, foi possível projetar um sistema de controle por modos deslizantes baseado em observadores da norma capaz de estabilizar globalmente o sistema em malha fechada mesmo considerando seus parâmetros incertos. Com o uso da técnica de observadores de norma, as estimativas da norma do estado usadas no projeto do controlador são maiores do que a do sistema original de Lorenz após uma fase transitória. Com isso, a amplitude do sinal de controle tende a diminuir a medida que o estado tende para zero. Além disso, estimativas menos conservadoras podem ser obtidas se o grau de incerteza no sistema diminuir, isto 


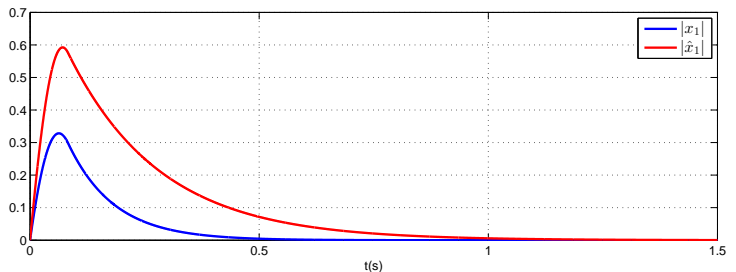

Figura 8: Comportamento da norma de $x_{1}$ e de $\hat{x}_{1}$, em malha fechada.

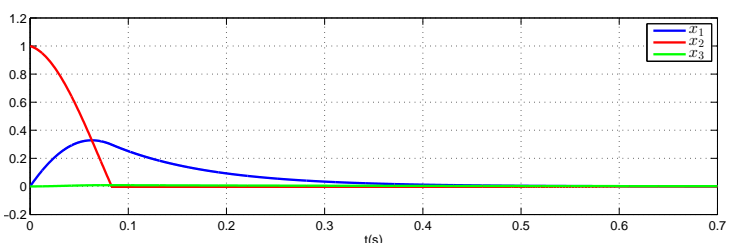

Figura 9: Comportamento das variáveis de estado $x_{1}, x_{2}$ e $x_{3}$, em malha fechada.

é, nos aproximar-se dos valores reais dos parâmetros. Por fim, a justificativa para colocar o sinal de controle no segundo canal do sistema (1)-(3) e a escolha da saída $y=x_{2}$ deve-se ao fato de que, apenas sobre estas condições, consegue-se garantir a não perda de controlabilidade do sistema, além de descrevermos todas as componentes do vetor de estado como subsistemas ISS com respeito a saída medida.

\section{Referências}

Araujo, A. D. and Singh, S. N. (2002). Output feedback adaptive variable structure control of chaos in lorenz system, International Journal of Bifurcation and Chaos 12(03): 571-582. DOI: 10.1142/S0218127402004516

Cheng-Yu Yeh, J. S. and Yau, H.-T. (2012). Circuit implementation of coronary artery chaos phenomenon and optimal pid synchronization controller design, Mathematical Problems in Engineering 2012.

Cuomo, K. M. and Oppenheim, A. V. (1993). Circuit implementation of synchronized chaos with applications to communications, Physical Review Letters $\quad \mathbf{7 1}(1)$ : $\quad 65-68 . \quad$ DOI: 10.1103/PhysRevLett.71.65

Filippov, A. (1964). Differential equations with discontinuous right-hand side., 42(2): 199-231.

Goodwin, R. M. (1990). Chaotic Economic Dynamics, Clarendon Press Oxford. DOI: 10.1093/0198283350.001.0001

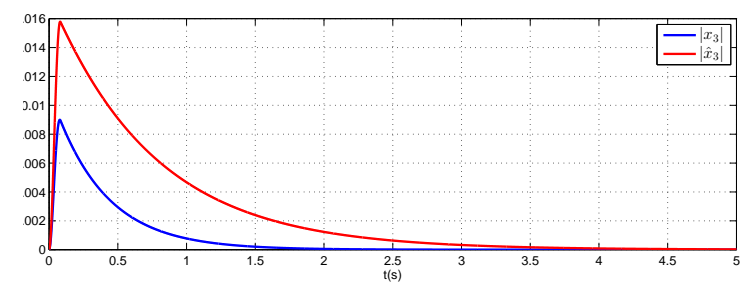

Figura 10: Comportamento da norma de $x_{3}$ e de $\hat{x}_{3}$, em malha fechada.
Guo, W. and Liu, D. (2011). Adaptive control of chaos in chua's circuit, Mathematical Problems in Engineering 2011.

Hou, Y.-Y. (2012). Controlling chaos in permanent magnet synchronous motor control system via fuzzy guaranteed cost controller, Abstract and Applied Analysis, Vol. 2012, Hindawi Publishing Corporation. DOI: 10.1155/2012/650863

Khalil, H. (2002). Nonlinear systems, Prentice hall Upper Saddle River.

Krichman, M., Sontag, E. D. and Wang, Y. (2001). Input-output-to-state stability, SIAM Journal on Control and Optimization 39(6): 1874-1928. DOI: $10.1137 /$ S0363012999365352

Lorenz, E. N. (1963). Deterministic nonperiodic flow, Journal Of The Atmospheric Sciences 20(2): $\quad 130-141$. DOI: 10.1175/1520-0469(1963)020<0130:DNF>2.0.CO;2

Mozelli, L. A., Campos, C. D., Palhares, R. M., Torres, L. A. B. and Mendes, E. M. A. M. (2007). Chaotic synchronization and information transmission experiments - a fuzzy relaxed h-infinity control approach., 1: 427-449.

Oliveira, T. R., Peixoto, A. J. and Hsu, L. (2010). Sliding mode control of uncertain multivariable nonlinear systems with unknown control direction via switching and monitoring function, IEEE Transactions on Automatic Control 55: 10281034. DOI: $10.1109 /$ TAC.2010.2041986

Ott, E., Grebogi, C. and Yorke, J. A. (1990). Controlling chaos, Physical Review Letters 64(11): 11961199. DOI: 10.1103/PhysRevLett.64.1196

Saltzman, B. (1962). Finite amplitude free convection as an initial value problem-i, Journal Of The Atmospheric Sciences 19(4): 329-341. DOI: 10.1175/1520-0469(1962)019<0329:FAFCAA>2.0.CO;2

Sontag, E. D. and Wang, Y. (1997). Output-to-state stability and detectability of nonlinear systems, Systems \& Control Letters 29(5): 279-290. DOI: 10.1016/S0167-6911(97)90013-X

Souza, F. O., Palhares, R. M., Mendes, E. M. A. M. and Torres, L. A. B. (2008). Robust h-infinity control for master-slave synchronization of lur'e systems with time-delay feedback, pp. 11611173.

Souza, F. O., Palhares, R. M., Torres, L. A. B. and Mendes, E. M. A. M. (2012). Synchronizing continuos time chaotic systems over nondeterministic networks with packet dropouts., 22.

Utkin, V., Guldner, J. and Shi, J. (1999). Sliding Mode Control in Electromechanical Systems, Taylor \& Francis Ltd.

Utkin, V. I. (1978). Sliding Modes And Their Application In Variable Structure Systems, Mir Publishers.

Wang, C. and Ge, S. S. (2001). Adaptive backstepping control of uncertain lorenz system, International Journal of Bifurcation and Chaos 11(04): 11151119 